\title{
Ischemia/Reperfusion Injury and Hepatocellular Carcinoma Recurrence After Liver Transplantation: Cancer at WIT's End?
}

\author{
Adrian H. Cotterell ${ }^{1,3}$ - Robert A. Fisher ${ }^{2}$
}

Published online: 2 June 2015

(c) Springer Science+Business Media New York 2015

Hepatocellular carcinoma (HCC) is the second most common cause of cancer deaths worldwide, with hepatitis B representing its major risk factor. In the Western world, the risk factors have included infection with the more prevalent hepatitis $\mathrm{C}$ virus and alcoholic cirrhosis. With effective therapy for hepatitis $\mathrm{C}$ now available and with the obesity epidemic on the rise, the relative contribution of nonalcoholic steatohepatitis (NASH) to the incidence of HCC will undoubtedly increase.

Management of HCC is challenging and complex, particularly in the setting of advanced liver disease. Liver transplantation has emerged as the treatment of choice for select patients with HCC and cirrhosis. Mazzaferro et al. [1] reported excellent survival in patients with cirrhosis complicated by HCC who were considered to be transplant candidates according to what is now known as the Milan criteria, "a tumor $5 \mathrm{~cm}$ or less in diameter in patients with single hepatocellular carcinomas and no more than three tumor nodules, each $3 \mathrm{~cm}$ or less in diameter, in patients with multiple tumors." Still, despite careful patient selection and the use of multimodality ablative therapy prior to transplantation, $\sim 12 \%$ of patients deemed suitable candidates according to the Milan criteria at the time of

Adrian H. Cotterell

acotterell@mcvh-vcu.edu

1 Hume-Lee Transplant Center, Medical College of Virginia Campus, Virginia Commonwealth University, Richmond, VA, USA

2 Transplant Institute, Beth Israel Deaconess Medical Center, Harvard Medical School, Boston, MA, USA

3 Transplant Surgery, Virginia Commonwealth University Health System, Richmond, VA, USA transplantation will experience recurrence of $\mathrm{HCC}$ within 5 years [2].

In this issue of Digestive Diseases and Sciences, Kornberg et al. [3] in an article entitled "Extended ischemia times promote risk of HCC recurrence in liver transplant patients" provide evidence that a prolonged warm ischemic time (WIT), the time taken to implant the liver allograft after its removal from cold storage, is an independent risk factor for recurrence of HCC after liver transplantation. In a retrospective analysis of 103 patients who underwent liver transplantation for HCC associated primarily with alcoholic and hepatitis $\mathrm{C}$ and $\mathrm{B}$ cirrhosis, the authors reported a recurrence rate of $12.7 \%$ for patients within the Milan criteria as opposed to $39 \%$ for those outside the criteria. In addition to the Milan criteria, Cox regression analysis identified serum $\alpha$-fetoprotein concentration $>400 \mathrm{IU} / \mathrm{ml}$ (a marker of tumor burden) [4], ${ }^{18} \mathrm{~F}$-fluorodeoxyglucose positron emission tomography (FDG PET)-positive tumors (signifying the presence of microvascular invasion and lack of tumor differentiation) [5], and WIT $>50 \mathrm{~min}$ as independent predictors of HCC recurrence. Of these, elevated serum $\alpha$ fetoprotein and PET-positive tumors are established risk factors for recurrence, as is HCC that exceeds the Milan criteria, whereas WIT $>50 \mathrm{~min}$ is a less well-documented risk factor for $\mathrm{HCC}$ recurrence in the setting of clinical liver transplantation.

In the subset of patients who met Milan criteria, tumor recurred in $42.2 \%$ with WIT $>50 \mathrm{~min}$ as opposed to $0 \%$ recurrence in patients with shorter WIT. In the subset of patients not meeting Milan criteria, $76.9 \%$ of patients with WIT $>50$ min developed recurrence compared with $22.5 \%$ of patients with WIT $<50$ min. In the subset of patients with PET-positive tumors, only WIT $>50 \mathrm{~min}$ was identified as an independent risk factor for HCC recurrence according to multivariate Cox regression analysis. 
In addition to occurring more frequently, $\mathrm{HCC}$ recurrences also occurred earlier in the WIT $>50 \mathrm{~min}$ cohort as compared to the WIT $<50$ min group. Nagai et al. [6], using a similar retrospective design, also identified WIT $>50 \mathrm{~min}$ as an independent risk factor for HCC recurrence after liver transplantation.

Why, then, is a prolonged WIT associated with tumor recurrence? Ischemic injury occurs during cold storage of livers, primarily to hepatic sinusoidal endothelial cells. Rewarming damages hepatic parenchymal cells, which, upon reperfusion, generate damage-associated molecular patterns (DAMPs) that serve as endogenous ligands for pattern recognition receptors (PRRs) such as Toll-like receptors (TLRs) expressed on non-parenchymal cells such as Kupffer cells [7]. Activated Kupffer cells produce inflammatory cytokines. This inflammatory response, which occurs ostensibly to recruit cells to repair tissue damage, may promote tumor recurrence in the setting of HCC.

Prolonged hepatic warm ischemia is complicated by hypoxia, ATP depletion, and other metabolic disturbances which perturb key intracellular organelles such as mitochondria and the endoplasmic reticulum (ER) leading to cellular stress responses such as the ER stress response. The lumen of the ER, an oxidative environment required for the proper folding of proteins, is disrupted by ischemia/ reperfusion injury, with resultant accumulation of unfolded proteins. The ER stress response includes the augmented expression of gene products that enhance protein folding capacity and the degradation of misfolded proteins. The priming of Kupffer cells to produce proinflammatory cytokines such as tumor necrosis factor (TNF)- $\alpha$ and interleukin (IL)-6 in response to TLR signaling after prolonged liver ischemia is augmented by the ER stress response [8]. ER stress thus constitutes a pro-inflammatory activation mechanism among innate immune cells in cooperation with TLR activation.

HCC can develop due to viral oncogenic transformation in the absence of cirrhosis as can occur with the hepatitis B virus. It also develops in the setting of chronic inflammation of the liver due to viral, alcoholic, or nonalcoholic etiologies. HCC can originate from adult hepatocytes as well as from hepatic progenitor cells. Pro-inflammatory cytokines such as TNF- $\alpha$ and IL-6 direct the dedifferentiation of tumor-derived hepatocyte-like cells into cancer progenitor cells [9].

Augmented TNF- $\alpha$ production occurring as a result of experimentally induced ER stress can be further increased with the consumption of a high-fat diet. The stress response is sustained when combined with hyper-nutrition resulting in an inflammatory environment in which NASH develops from simple steatosis. Oxidative stress leads to genomic instability and oncogenic mutations. TNF- $\alpha$ produced by activated inflammatory macrophages stimulates compensatory hepatocyte proliferation to include the HCC progenitors [10].

In the setting of liver transplantation for $\mathrm{HCC}$, circulating cancer progenitor cells may encounter a pro-oncogenic proinflammatory environment in the setting of the reperfused liver allograft. Prolonged WIT sustains ER stress while increasing production of TNF- $\alpha$ and other proinflammatory cytokines by the Kupffer cells and other non-parenchymal cells. TNF- $\alpha$ may then stimulate the expansion of the circulating cancer progenitor cells with resultant tumor recurrence. The goal of multimodality ablative therapy of HCC prior to liver transplantation should thus be the eradication of all viable tumor cells, eliminating any substrate for tumor growth in the pro-oncogenic, pro-inflammatory environment of the reperfused liver. WIT may not be easily manipulated, but its downstream effects may be modulated. If circulating cancer progenitor cells persist, attempts to ameliorate ischemia/reperfusion injury of the liver allograft such as TLR blockade or anti-TNF- $\alpha$ agents may help prevent the recurrence of HCC after liver transplantation.

\section{References}

1. Mazzaferro V, Regalia E, Doci R, et al. Liver transplantation for the treatment of small hepatocellular carcinomas in patients with cirrhosis. N Engl J Med. 1996;334:693-699.

2. Ramanathan R, Sharma A, Lee DD, et al. Multimodality therapy and liver transplantation for hepatocellular carcinoma: a 14-year prospective analysis of outcomes. Transplantation. 2014;98:100-106.

3. Kornberg A, Witt U, Kornberg J, et al. Extended ischemia times promote risk of HCC recurrence in liver transplant patients. Dig Dis Sci. (Epub ahead of print). doi:10.1007/s10620-015-3541-z.

4. Hsu C, Liu P, Lee Y, et al. Using serum $\alpha$-fetoprotein for prognostic prediction in patients with hepatocellular carcinoma: what is the most optimal cutoff? PLoS One. 2015;10:e0118825.

5. Lee S, Kim S, Kim S, et al. Clinical impact of ${ }^{18} \mathrm{~F}$ fluorodeoxyglucose positron emission tomography/computed tomography in living donor liver transplantation for advanced hepatocellular carcinoma. Transplantation. 2015. doi:10.1097/ TP.0000000000000719.

6. Nagai S, Yoshida A, Facciuto M, et al. Ischemia time impacts recurrence of hepatocellular carcinoma after liver transplantation. Hepatology. 2015;61:895-904.

7. Zhai Y, Petrowsky H, Hong J, et al. Ischemia-reperfusion injury in liver transplantation-from bench to bedside. Nat Rev Gastroenterol Hepatol. 2013;10:79-89.

8. Rao J, Yue S, Fu Y, et al. ATF6 mediates a pro-inflammatory synergy between ER stress and TLR activation in the pathogenesis of liver ischemia-reperfusion injury. Am J Transplant. 2014;14:1552-1561

9. Dubois-Pot-Schneider H, Fekir K, Coulouarn C, et al. Inflammatory cytokines promote the retrodifferentiation of tumorderived hepatocyte-like cells to progenitor cells. Hepatology. 2014;60:2077-2090.

10. Nakagawa H, Umemura A, Taniguchi K, et al. ER stress cooperates with hypernutrition to trigger TNF-dependent spontaneous HCC development. Cancer Cell. 2014;26:331-343. 